\title{
Estado de conservación del complejo cenagoso La Larga a partir del análisis ecológico de la avifauna, enfatizando en especies endémicas, migratorias, amenazadas y susceptibles al tráfico
}

\section{Status conservation of La Larga wetlands complex based on ecological analysis of birdlife present emphasizing in endemic, migratory, threatened an cites species}

\section{Nelsy Sofía Bonilla-Urrutia*}

\section{Resumen}

Se presenta la caracterización de la avifauna observada en los diferentes estratos del complejo cenagoso La Larga, como el espejo de agua, vegetación sumergida y bosque circundante, para lo cual se realizaron muestreos diarios (mañana y tarde), aplicando una evaluación rápida mediante observaciones directas, además de entrevistas informales a cazadores. Se registraron 40 especies de aves pertenecientes a 22 familias y 15 órdenes. Las familias más representativas fueron Accipitridae y Ardeidae. La mayor ocurrencia de individuos (aves acuáticas y semiacuáticas) se registró en la vegetación sumergida. El chamón (Crotophaga major), fue la especie más abundante del complejo. Se identificaron 5 gremios tróficos, siendo los carnívoros con 33\% los más representativos; estos actúan como indicadores del buen estado trófico del ambiente. Se identificó una especie amenazada y dos con susceptibilidad al tráfico, además de cuatro especies casi endémicas y cuatro migratorias. Estas le confieren al ecosistema gran relevancia, convirtiéndolo en una importante área de conservación.

Palabras clave: Avifauna; Ciénaga; Complejo cenagoso La Larga; Tagachí.

\begin{abstract}
One presents the characterization of the avifauna observed in the different strata of the wetlands complex La Larga, as the mirror of water, plunged vegetation and surrounding forest, for which there were realized daily samplings (tomorrow and the evening), applying a rapid evaluation by means of direct observations, besides informal interviews to hunters. There were registered 40 species of birds belonging to 22 families and 15 orders. The most representative families were Accipitridae and Ardeidae. The major occurrence of individuals (aquatic and semiaquatic birds) registered in the plunged vegetation. The chamón (Crotophaga major), was the most abundant species of the complex; 5 unions were identified tróficos, being the carnivorous ones with 33 most representative \%; these act as indicators of the good condition trófico of the environment. A threatened species and two were identified with suceptibilidad to the traffic, besides four almost endemic species and migratory four. These award to the ecosystem great relevancy, turning it into an important area of conservation.
\end{abstract}

Keywords: Birdlife; Wetlands; Wetlands complex; La Larga; Tagachi.
Bióloga, Contratista Instituto de Investigaciones Ambienales de Pacífico (IIAP), Quibdo, Colombia.

e-mail: sofibon@gmail.com

Recibido: 31 de enero de 2012

Aceptado: 3 de abril de 2012 


\section{Introducción}

A nivel faunístico las ciénagas son consideradas ecosistemas altamente productivos, que permiten el flujo de la energía a través de niveles tróficos complejos, que van desde macroinvertebrados acuáticos hasta aves carroñeras eliminadoras de la materia orgánica, que terminan devolviéndola al ecosistema como parte de una dinámica ecológica que incluye dos ambientes totalmente distintos, que responden a los requerimientos de los organismos existentes. La fauna presente en estos ecosistemas hace parte esencial en la funcionalidad del mismo y en la composición del paisaje, recreándolo y ayudando a su mantenimiento en el tiempo y el espacio.

La fauna silvestre de los humedales es quizá, una de las más ricas y variadas de los ecosistemas del planeta. Los innumerables microhábitats, la enorme productividad primaria, los nutrientes que por él circulan, ayudan a explicar esta inestimable diversidad. Los animales que habitan los humedales tienen diversos mecanismos para adaptarse a ellos. Patas palmeadas con membradas interdigitales para nadar, picos aguzados como las garzas para proveerse el alimento, plumas y pelos muy bien lubricados para protegerse del frío y muchas aves de picos aplanados para filtrar alimento.

Las anteriores razones crean la necesidad de conocer el potencial faunístico de las ciénagas y el papel que las especies desempeñan en la funcionalidad y mantenimiento de dicho ecosistema. Esta investigación tiene como objetivo caracterizar y analizar el estado de conservación de la avifauna presente en el complejo cenagoso La Larga, a partir del conocimiento de su composición, además de la identificación de especies relevantes (migratorias, endémicas, amenazadas y susceptibles al tráfico), claves para dicho funcionamiento y conservación de este ecosistema.

Área de estudio. El presente estudio se llevó a cabo en el corregimiento de Tagachí (Quibdó), del cual hace parte el complejo cenagoso La Larga. Este complejo se localiza en la parte media del río Atrato, la cual pertenece a una zona con balance hídrico húmedo a muy húmedo (112 $\mathrm{mm}$ a $248 \mathrm{~mm})$ y se encuentra incluido en la unidad de llanuras aluviales $(0$ a $50 \mathrm{msnm}$ ) (Rangel 2004). Todos los suelos de la zona son de vocación forestal y el desarrollo de los cultivos autóctonos con sus mecanismos específicos de adaptación, pueden aumentar su productividad. Las subcuencas de la margen izquierda están representadas por los ríos Beté, Bojayá, Aibí, Buchadó, Buey, Munguidó, Tagachí y Tanguí.

Tagachí, es en términos generales un corregimiento con vocación pesquera y forestal, con numerosos ecosistemas de ciénagas como la Rinconera, La Larga y Anchuarrá entre otras. Sus tierras son aptas para el desarrollo de diversas actividades productivas.
Descripción del área de muestreo. Se hicieron observaciones en distintos hábitats dentro del área de estudio, las cuales fueron seleccionadas con el propósito de caracterizar los diferentes estratos presentes en el ecosistema como el espejo de agua, la vegetación sumergida y el bosque circundante (tierra firme).

La vegetación sumergida se encuentra conformada por especies herbáceas y arbustivas enraizadas y algunas arbóreas que rodean el borde del humedal, las cuales toleran bien el agua y proveen hábitat y alimento a la avifauna residente y visitante del lugar. Se encuentran algunas especies productoras de frutos y que sirven de percha para aves de pequeño, mediano y gran porte, además de algunas plantas cargadas de bromelias en estado de floración. También se presentan matorrales que son utilizados como sitio de percha para pequeñas aves acuáticas que se posan para visualizar su presa y para el descanso. La composición de dicha vegetación varía a lo largo del borde de la ciénaga (Figura 1).

El bosque circundante se caracteriza por ser un área altamente intervenida, donde es notable la extracción forestal que ha producido algunos claros, los cuales son aprovechados por especies cuyos requerimientos ecológicos exigen áreas abiertas y la influencia directa del brillo solar, como es el caso de lagartos que son la presa preferida de aves carnívoras; se observan especies de palmas y árboles de gran porte (de hasta 30 metros de altura) que proporcionan una importante oferta trófica (frutos) para aves como las guacamayas y tucanes que obtienen el alimento necesario para su supervivencia; estas especies arbóreas sirven de percha para el descanso temporal de la fauna avícola; muchos de estos árboles portan bromélias en floración que sirven como hábitat y fuente de alimento para anfibios, insectos y aves nectarívoras; además, hay variedad de especies arbustivas productoras de frutos en baya aprovechados por aves frugívoras (Figura 2).

También se hicieron observaciones en el caño que conecta la ciénaga y el río Atrato y en zonas de cultivo aledañas al humedal, donde se encuentran cultivos de arroz, borojó, guayaba agria, limón y maíz, los que además de generar la producción agrícola de habitantes de la zona, constituyen la oferta alimenticia de algunas especies de aves que aprovechan tal recurso.

\section{Métodos}

Entrevistas dirigidas a la comunidad. Se realizaron entrevistas informales a cazadores, los cuales ayudaron a identificar con la utilización de guías ilustradas, la existencia de aves en el lugar, permitiendo la recopilación de información sobre la productividad del ecosistema y de las comunidades avícolas que allí habitan.

Muestreos. El levantamiento de la información se realizó 


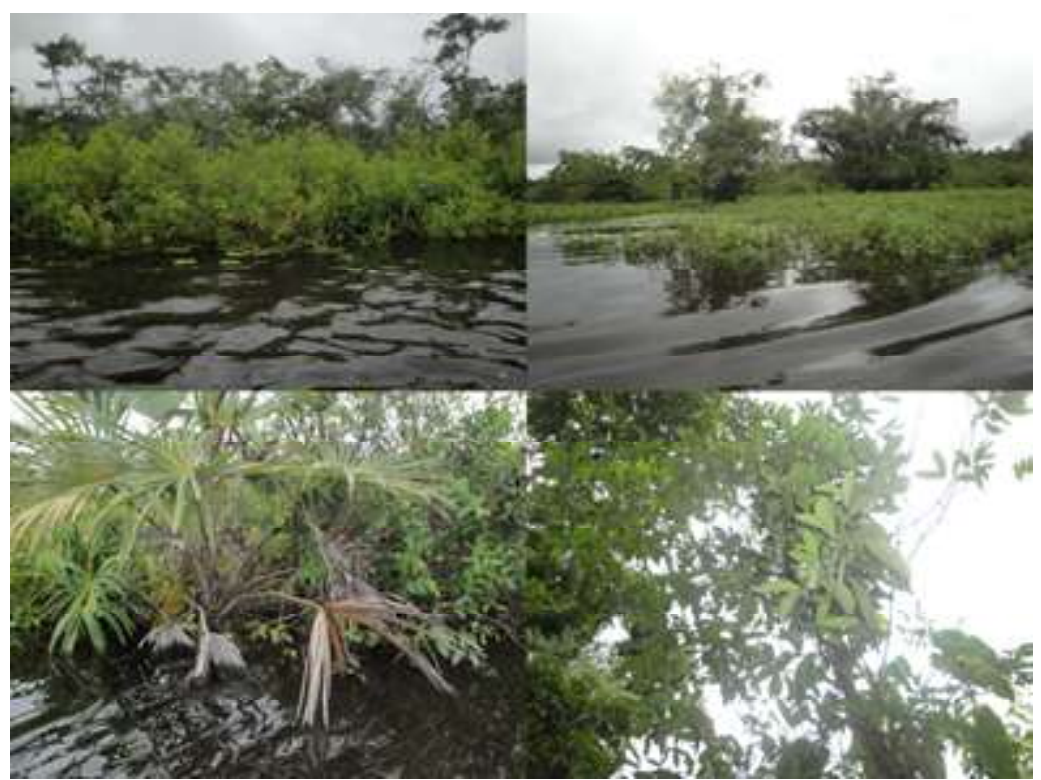

Figura 1. Vegetación sumergida que rodea el complejo cenagoso

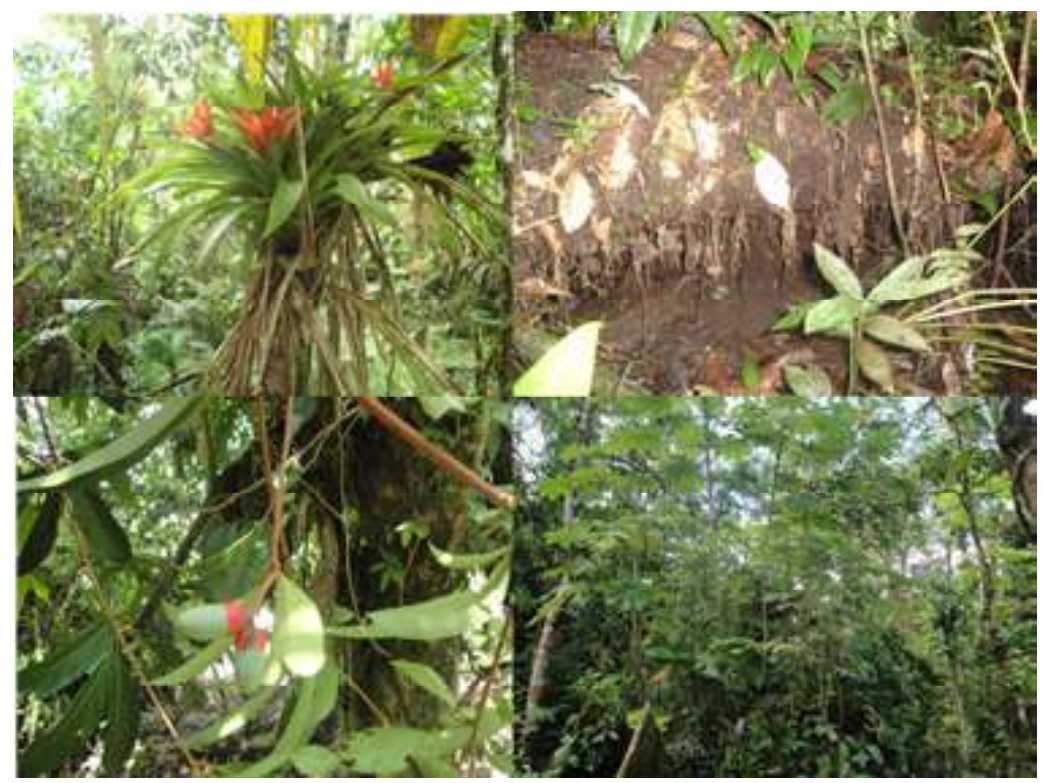

Figura 2. Oferta de recursos presentes en el bosque adyacente al complejo La Larga

mediante la implementación de muestreos diarios durante la mañana y la tarde, aplicando una intensidad de 2 horas en cada período. Las observaciones se realizaron en diferentes microambientes como el espejo de agua, la vegetación sumergida, caños o meandros aledaños al humedal y vegetación adyacente al mismo. En todos los puntos se aplicó una evaluación rápida de la avifauna TNC?? (2000). Se empleó el método de observación directa utilizando binoculares marca Russell (10 X 40), se tuvo en cuenta las características morfológicas del individuo (coloración, forma del pico y cola) y fueron comparados e identificados con las guías de campo ilustradas de aves de Hillty y Brown (2001), Roda et al. (2003), Rodríguez y Hernández (2002) y McMullan et al. 2010; algunos de los individuos se fotografiaron para una mejor identificación.

\section{Resultados y discusión}

Composición de la ornitofauna. Se registraron 40 especies de aves pertenecientes a 22 familias y 15 órdenes. De estas, 17 se observaron y 22 se reportaron por cazadores habitantes de la comunidad de Tagachí. Los órdenes Pelecaniformes, Accipitriformes, Charadriiformes fueron los más representativos con 10 , 8 y 5 especies respectivamente. Las familias que registraron mayor representatividad fueron Accipitridae y Ardeidae con 5 especies cada una, seguidas por Alcedinidae, Pelecanidae y Cracidae con 3 especies cada una (Figura 3).

La riqueza de aves registrada en este estudio difiere significativamente de la reportada por el IIAP (2008), que registra para la ciénaga Grande de Beté 60 especies, lo cual se atribuye a la presencia, en esta última, de mayor cantidad de macrófitas en el espejo de agua, y por lo tanto gran abundancia de macroinvertebrados y peces, recurso trófico de la mayoría de aves que habitan o visitan estos ecosistemas, además de la presencia de una mayor continuidad del bosque, con lo que se facilita el movimiento de las aves a lo largo del complejo; estas condiciones se presentan en menor intensidad en el complejo La Larga, lo que posiblemente pudo incidir en los valores de riqueza allí obtenidos.

No obstante, el complejo cenagoso La Larga hace un gran aporte a la diversidad gamma de aves acuáticas en la cuenca del Atrato, lo que se debe al recambio de especies (diversidad beta) favorecido por la variedad de hábitats y la oferta de recursos que exhiben estos ecosistemas; esto fue verificado durante los muestreos, pudiéndose observar una gran cantidad de árboles frutales (en su mayoría churimo) en el borde del bosque y hacia el interior del mismo, especies como el madroño y variedad de palmas productoras de semillas muy apetecidas para aves como loros y tucanes, además de la presencia de una variedad de peces que sirven como alimento para muchas 


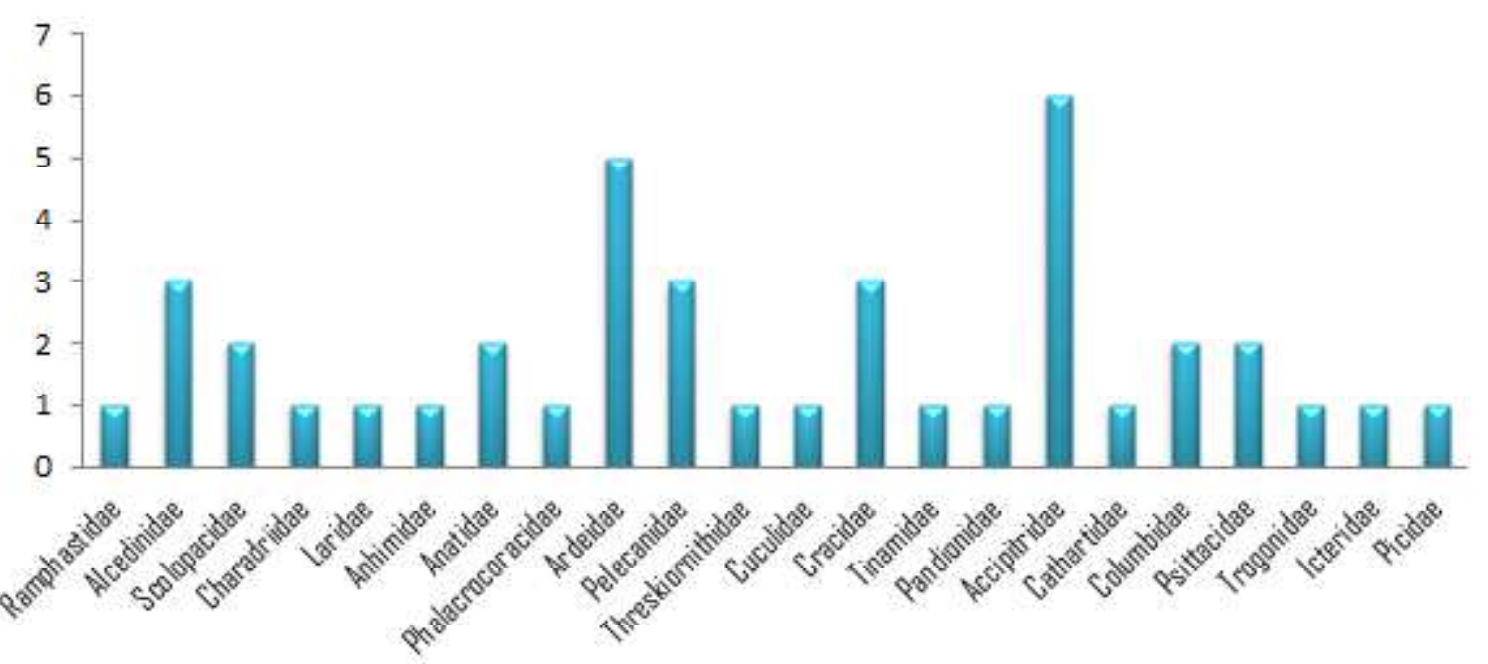

Figura 3. Número de especies por familias que componen la ornitofauna del
complejo cenagoso La Larga

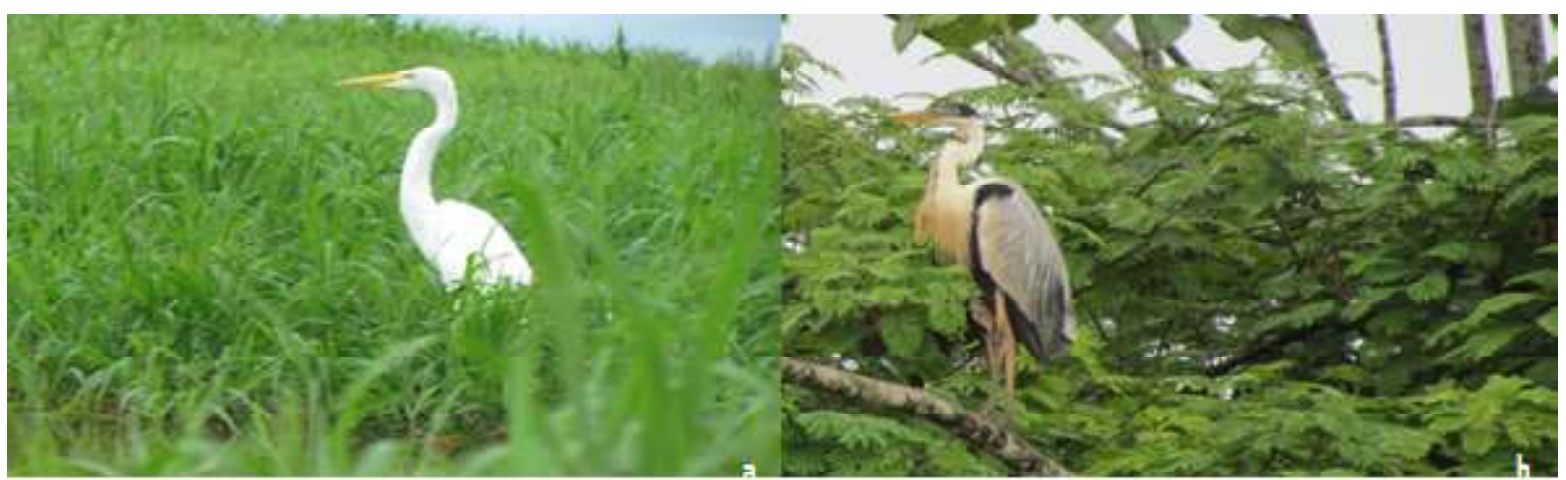

Figura 4. Aves perchando en la vegetación que rodea la ciénaga: a. Ardea alba en pastizal, b. A. cocoi en el matorral

aves piscívoras.

Especies asociadas con diferentes hábitats del complejo. De las 19 especies observadas, 10 se registraron en el bosque circundante, 9 en vegetación flotante, 6 en el caño y 2 en el espejo de agua; 6 de ellas se encontraron en diferentes hábitats. La mayor ocurrencia de individuos se registró en la vegetación flotante, siendo en su mayoría aves acuáticas y semiacuáticas que buscan alimento en el espejo de agua y que utilizan dicha vegetación como sitio de percha. También se encontraron especies frugívoras en el bosque circundante que aprovechan la oferta alimenticia (frutos) proporcionada por los árboles y arbustos en fructificación que conforman esta zona. Además se registraron especies de aves silvícolas que se alimentan básicamente de frutos y semillas, oferta altamente observada en los recorridos (Tabla 1).

Dentro de las especies registradas cabe destacar gran cantidad de garzas (familia Ardeidae), que se encuentran bien representadas por especies como Ardea cocoi, A. alba,
Butorides striata, Egretta tricolor y Bubuculus ibis, que son exclusivas de ecosistemas acuáticos. Algunas de estas fueron observadas con frecuencia a lo largo de la ciénaga y en los caños, lo que indica el buen estado de las poblaciones de otras especies como peces, moluscos y crustáceos, entre otros, que son la base alimenticia de esta familia (Figura 4). También se pudo observar en la vegetación sumergida y en el caño, miembros de la familia Alcedinidae, que estuvo representada por tres especies de Martín pescador (Chloroceryle amazona, C. americana y Megaceryle torcuata) en moderada abundancia, debido a la oferta piscícola y de insectos acuáticos presentes en el ecosistema. Los remanentes de bosque adyacentes al humedal, así como el interior del bosque, fueron los hábitats propicios para el registro de aves silvícolas como guacamayas (Ara severus y Ara araunana) y tucanes (Ramphastos brevis).

El chamón (Crotophaga major), fue la especie más abundante del complejo, siendo la más conspicua en el área; 
Bioetnia Volumen 9 № 1 (enero-junio), 2012

Tabla 1

Distribución de la avifauna observada en los diferentes tipos de hábitats del complejo cenagoso La Larga

\begin{tabular}{|c|c|c|c|c|}
\hline \multirow[t]{2}{*}{ Especies } & \multicolumn{3}{|c|}{ Hábitats } & \multirow[b]{2}{*}{ Caño } \\
\hline & Vegetación sumergida & Bosque circundante & Espejo de agua & \\
\hline Ramphastos brevis & & $\mathrm{x}$ & & \\
\hline Chlorocerile amazonas & $x$ & & & $x$ \\
\hline Chloroceryle americana & $x$ & & & $x$ \\
\hline Megaceryle torquata & $x$ & & & \\
\hline Jacana jacana & $x$ & & & \\
\hline Charadrius collaris & & & & $x$ \\
\hline Vanellus chilensis & & $x$ & & \\
\hline Chauna chavaria & $\mathrm{x}$ & & & \\
\hline Phalacrocorax brasilianus & & & $x$ & \\
\hline Ardea cocoi & $x$ & & $\mathrm{x}$ & $x$ \\
\hline Butorides striata & $x$ & & & $\mathrm{x}$ \\
\hline Crotophaga major & $x$ & $x$ & & $x$ \\
\hline Accipiter superciliosus & & $\mathrm{x}$ & & \\
\hline Ascipiter striatus & & $\mathrm{x}$ & & \\
\hline Cathartes aura & $\mathrm{x}$ & $x$ & & \\
\hline Zenaida asiatica & & $x$ & & \\
\hline Ara araunana & & $\mathrm{x}$ & & \\
\hline Cacicus cela & & $x$ & & \\
\hline
\end{tabular}

fue observada en los diferentes escenarios muestreados, por lo general en grupos perchados en árboles, pero también se observó ocasionalmente un individuo en vuelo o posado en los matorrales sumergidos. La variedad de Accipitridos como gavilán (Accipiter superciliosus), capes baja ( $A$. striatus), águila iguanera (Spizaetus tyrannus), caracolero (Rostrhamus sociabilis) y gavilán lagartero (Harpagus bidentatus), se debe quizás a que este ecosistema le brinda a estas aves gran variedad de sitios para la nidación y la reproducción, así como también provee de recurso trófico que va desde peces hasta primates; por lo tanto estas especies aprovechan los recursos disponibles en los distintos microambientes del lugar, lo que hace de la ciénaga un espacio visitado con frecuencia o habitado por especies carnívoras que actúan como controladores biológicos y que contribuyen con la diversidad y buen estado trófico del ambiente. Lo anterior es corroborado por Márquez et al. (2005) quien confirma el importante papel que cumplen estas aves en el ecosistema debido a que ocupan el último nivel de la cadena trófica. Burnham et al. (1989) por su parte, afirma que los requerimientos ecológicos de las aves rapaces ( $\mathrm{su}$ dieta carnívora entre otros) hacen que este grupo sea un excelente indicador biológico, por lo que su ausencia puede significar grandes cambios en los lugares donde suelen habitar. Además, estas al igual que otros depredadores, son grupos claves en los ecosistemas a los que pertenecen.

Los registros obtenidos del pato cuervo fueron escasos, observándose sólo tres individuos durante los muestreos. Una situación diferente fue reportada en la ciénaga Grande de Beté (IIAP 2008), donde esta fue la especie más abundante durante el inventario, obteniéndose registros de manera individual y en bandadas, en actividad de pesca y en descanso. Esta diferencia puede deberse a que en estos complejos la especie cuenta con áreas más amplias que le ofrecen mayor cantidad y variedad de hábitats y de alimento, porque presenta un amplio rango de movimiento, mostrando gran preferencia por este tipo de ambientes.

Composición trófica. Se identificaron 5 gremios tróficos distribuidos entre las 40 especies de aves registradas para la zona. El gremio más representativo en el área fue el de los carnívoros con 14 especies (33\%), seguido por el gremio de las aves con hábitos omnívoros con 8 especies $(21 \%)$, piscívoros con 7 (18\%), insectívoras con $6(15 \%)$ y en menor representatividad se encuentran los frugívoros con 5 espe- 
cies (13\%). A diferencia de la composición trófica registrada en este estudio, donde se ubica a los carnívoros como el gremio con mayor representatividad, los resultados de investigaciones como la realizada por Castaño (2001) en los humedales de la Guajira, muestra el grupo de las aves insectívoras como el gremio que ocupa el mayor porcentaje con el $48.5 \%$ de las aves registradas. Lo que puede estar relacionado con las diferencia de complejidad trófica de los dos lugares.

La presencia de aves carnívoras en el complejo cenagoso La Larga indica la existencia de gran variedad de presas, lo cual se traduce en una buena estratificación trófica del ecosistema. Especies como Butorides striata, Ardea alba, A. cocoi, así como también los alcedínidos (Tabla 2), que fueron observadas durante los muestreos, son indicadores de la presencia de peces que constituyen la presa principal de estas especies. En el caso de otras especies como Accipiter superciliosus y A. striatus, quienes incluyen en su dieta aves de pequeño tamaño, por lo general presentes en el dosel del bosque, son una muestra de la actividad trófica que se presenta en todos los estratos de este ecosistema. El éxito del gremio de los omnívoros en este ambiente está dado por su gran estrategia trófica generalista, presentando una amplia distribución en los diferentes estratos del ecosistema, encontrando gran variedad de alimento como forraje, frutos, invertebrados acuáticos y terrestres, peces, y vertebrados, además de un número variado de hábitats. La representatividad del grupo de insectívoros (Tabla 2) encontrados en el complejo se puede deber a que en estos ecosistemas las condiciones climáticas y el medio acuático como tal, favorecen la presencia de insectos y sus procesos reproductivos, lo cual hace que sean abundantes constantemente y provean de nutrientes a esta fauna avícola. Verea et al. (2000), afirman que este tipo de ambientes constituye una fuente importante de nutrientes ricos en proteínas y carbohidratos para las aves insectívoras, del mismo modo Castaño (2001), argumenta que estos organismo por lo general ocupan una amplia variedad de nichos y son abundantes a través de todos los estados sucesionales de la vegetación, cuerpos de agua y áreas altamente perturbadas y transformadas.

El gremio de los frugívoros es uno de los más importantes a nivel ecológico, porque involucra especies con una función relevante en el ecosistema, las cuales se desempeñan como dispersoras que facilitan el mantenimiento, reconstrucción y continuidad de la estructura vegetal y por lo tanto del paisaje. Se reportaron psitácidos, pavas, tucanes y pajuil (Tabla 2), que tienen gran preferencia por las semillas de palmas, recurso abundante en el complejo

\section{Especies de interés especial}

Amenazadas. Se registró la pava del Baudó (Penelope orthoni) como la única especie con amenaza de riesgo a la extinción; la Unión Internacional para la Conservación de la Naturaleza (UICN) (Rengifo et al. 2002) la categoriza como especie vulnerable (VU) presentándose como sus principales amenazas la deforestación y la consecuente pérdida del hábitat (Stotz et al. 1996), además se encuentra fuertemente presionada por la cacería. El Convenio sobre el Comercio Internacional de Especies Amenazadas de Flora y Fauna Silvestre (CITES) reporta en el Apéndice III como especie susceptible al tráfico a Crax rubra.

Migratorias. La comunidad reporta la visita ocasional de 6 especies de aves acuáticas, entre estas se encuentran la gaviota gris (Leucophaeus modestus), pato collarejo (Aythya collaris) y garza tricolor (E. tricolor), consideradas a nivel nacional como aves migratorias; estas visitas se realizan en verano, temporada que coincide con la reproducción activa de varias especies de peces, razón por la cual no se observaron durante los muestreos.

Endémicas. Se identificaron 4 especies casi endémicas de Colombia, Crypturellus kerrie, P. ortoni, Chauna chavaria y Ramphastos brevis, las cuales comparten su distribución con otros países de Suramérica. P. ortoni, además se considera una especie con rango de distribución restringido para la subregión del Chocó (Stiles 1998) (Tabla 3).

\section{Conclusiones}

El complejo cenagoso La Larga presenta una importante diversidad avícola, representada por una riqueza de 40 especies asociadas con ecosistemas húmedos, que involucra algunas acuáticas, semiacuáticas, playeras y silvícolas. Esto indica el buen estado del ambiente en términos de existencia de alta oferta trófica y variedad de elementos disponibles que actúan como hábitats y refugios temporales; además de condiciones hidrológicas y fisicoquímicas que permiten el mantenimiento y desarrollo de estas especies, las que a su vez presentan estrecha relación con una variada y compleja estructura vegetal que responde a los cambios hidrológicos temporales propios de un ecosistema inundable. Adicionalmente, los altos valores de riqueza de estos grupos faunísticos demuestran que a pesar de los niveles de intervención antrópica que presenta el ecosistema, aún existen elementos que sostienen grupos con específicos requerimientos ecológicos como es el caso de los halcones, que fueron avistados en el área, corroborando la indudable presencia de estas especies en el complejo La Larga.

Especies de aves como Butorides striata, Ardea cocoi, Chloroceryle americana, C. amazonas y Crotophaga major entre otras, se registraron con frecuencia durante los muestreos, siendo C. major la más abundante, afirmaciones que muestran a las aves carnívoras y omnívoras como las de mayor éxito en términos de abundancia, además, de actuar 
Bioetnia Volumen 9 No 1 (enero-junio), 2012

Tabla 2

Familias y especies distribuidas en gremios tróficos presentes en el complejo La Larga

\begin{tabular}{|c|c|c|}
\hline Gremio & Familia & Especie \\
\hline \multirow[t]{20}{*}{$\overline{\text { Carnívoros }}$} & Alcedinidae & Chlorocerile amazonas \\
\hline & & Chloroceryle americana \\
\hline & & Megaceryle torquata \\
\hline & Laridae & Leucophaeus modestus \\
\hline & Anatidae & Aythya collaris \\
\hline & & Anas strepera \\
\hline & Phalacrocoracidae & Phalacrocorax brasilianus \\
\hline & Ardeidae & Ardea cocoi \\
\hline & & Ardea alba \\
\hline & & Butorides striata \\
\hline & & Bubulcus ibis \\
\hline & Pelecanidae & Pelecanus occidentalis \\
\hline & & Fregata magnificens \\
\hline & Accipitridae & Accipiter superciliosus \\
\hline & & Ascipiter striatus \\
\hline & & Spizaetus tyrannus \\
\hline & & Rostrhamus sociabilis \\
\hline & & Harpagus bidentatus \\
\hline & Cathartidae & Cathartes aura \\
\hline & Pandionidae & Pandion haliaetus \\
\hline \multirow[t]{8}{*}{ Omnívoros } & Pelecanidae & Phoenicopterus ruber \\
\hline & Cuculidae & Crotophaga major \\
\hline & & Ortalis cinereiceps \\
\hline & Tinamidae & Crypturellus kerrie \\
\hline & Columbidae & Columbia livia \\
\hline & & Zenaida asiatica \\
\hline & Icteridae & Cacicus cela \\
\hline & Picidae & Dryocopus lineatus \\
\hline \multirow[t]{6}{*}{ Insectívoros } & Trogonidae & Trogon chionurus \\
\hline & Scolopacidae & Jacana jacana \\
\hline & & Charadrius collaris \\
\hline & Charadriidae & Vanellus chilensis \\
\hline & Ardeidae & Egretta tricolor \\
\hline & Threskiornithidae & Mesembrinibis cayennensis \\
\hline \multirow[t]{6}{*}{ Frugívoros } & Anhimidae & Chauna chavaria \\
\hline & Ramphastidae & Ramphastos brevis \\
\hline & Cracidae & Crax rubra \\
\hline & & Penelope ortoni \\
\hline & Psittacidae & Ara araruana \\
\hline & & Ara severus \\
\hline
\end{tabular}


Tabla 3

Especies de aves de interés especial presentes en el complejo cenagoso La Larga

\begin{tabular}{|c|c|c|c|c|c|}
\hline \multirow[t]{2}{*}{ Orden } & \multirow[t]{2}{*}{ Familia } & \multirow[t]{2}{*}{ Nombre científico } & \multicolumn{2}{|c|}{ Estado de conservación } & \multirow[t]{2}{*}{ END/MIG } \\
\hline & & & UICN & CITES & \\
\hline \multirow[t]{2}{*}{ Piciformes } & Ramphastidae & Ramphastos brevis & & & $\mathrm{CE}$ \\
\hline & Laridae & Leucophaeus modestus & & & M \\
\hline \multirow[t]{6}{*}{ Anseriformes } & Anhimidae & Chauna chavaria & & & $\mathrm{CE}$ \\
\hline & Anatidae & Aythya collaris & & & $M$ \\
\hline & & Egretta tricolor & & & M \\
\hline & Pelecanidae & Pelecanus occidentalis & & & v \\
\hline & & Phoenicopterus ruber & & & v \\
\hline & & Fregata magnificens & & & v \\
\hline \multirow[t]{2}{*}{ Galliformes } & Cracidae & Crax rubra & & III & \\
\hline & & Penelope ortoni & VU & & CE-DR. \\
\hline Tinamiformes & Tinamidae & Crypturellus kerrie & & & CE \\
\hline \multirow[t]{3}{*}{ Accipitriformes } & Pandionidae & Pandion haliaetus & & & $\mathrm{Mb}$ \\
\hline & & Harpia harpyja & & I & \\
\hline & & Zenaida asiatica & & & v \\
\hline
\end{tabular}

$\mathrm{V}$ (visitante), M (migratoria), Mb (migratoria boreal), CE (casi endémica), CE-DR (casi endémica con distribución restringida).

como indicadores potenciales de la presencia de oferta trófica compuesta por peces, insectos y frutos. Los altos registros de especies carnívoras $33 \%$ indican la presencia de especies que actúan como presa de los mismos y que sostienen sus poblaciones, lo que constituye una red trófica bien estratificada. También fue evidente la presencia de variedad de aves frugívoras, que actúan como dispersoras de semillas, con lo que contribuyen significativamente con la continuidad y funcionalidad del bosque.

Se identificó la presencia de una especie de ave amenazada y dos especies con susceptibilidad al tráfico, además de cuatro especies casi endémicas y cuatro migratorias. Todas estas son de gran interés para la conservación y le confieren a este ecosistema gran relevancia, al punto de convertirlo en una importante área de conservación que requiere el adecuado manejo de los recursos biológicos allí presentes.

\section{Literatura citada}

Burnham, W. A., J. P. Jenny, C. W. Turley (eds.). 1989. Progress Report II, Maya Project. Use of raptors as environmental indicators for design and management of protected areas and for building local capacity in Latin America. Boise: The Peregrine Fund.

Cataño, G. J. 2001. Evaluación de la avifauna asociada a humedales costeros de la guagira con fines de conservación. Crónica forestal y del medio ambiente 16 (1): 5-33.
CITES y UNEP. 2010. Convención sobre el Comercio Internacional de Especies Amenazadas. Apéndices I, II y III. Disponible en http:// www.cites.org

Hilty, S. L., W. L. Brown. 1986. A guide to the birds of Colombia. Princeton: Princeton University Press. 836 pp.

Instituto de Investigaciones Ambientales del Pacífico. 2008. Inventario, priorización y caracterización de las ciénagas del municipio del Medio Atrato, Chocó. Informe final. Quibdó; IIAP. $195 \mathrm{pp}$.

McMullan, M., A. Quevedo, T. Donegan. 2010. Guía de campo de las aves de Colombia. Bogotá; ProAves.

Márquez, C., M. Bechard, F. Gast, V.H. Vanegas. 2005. Aves rapaces diurnas de Colombia. Bogotá: Instituto de Investigación de Recursos Biológicos «Alexander von Humboldt». 394 pp.

Rangel-Ch., J. O. 2004. Colombia diversidad biótica. IV. El Chocó biogeográfico: ambiente fisico. Bogotá: Instituto de Ciencias Naturales, Universidad Nacional de Colombia.

Rengifo, L. M., A. M. Franco-Maya, J. D. Amaya-Espinel, G. H. Kattan, B. López-Lanus, (eds.). 2002. Libro Rojo de Aves de Colombia. Serie de Libros Rojos de Especies Amenazadas de Colombia. Bogotá: Instituto de Investigación de Recursos Biológicos «Alexander von Humboldt» y Ministerio del Medio Ambiente.

Rodá, F., J. Ibáñez, C. Gracia. 2003. L'estat dels boscos. En: L'estat del Medi Ambient Catalunya. Catalunya: Generalitat de Catalunya.

Rodríguez, J., J. Hernández. 2002. Loros de Colombia. Serie de Guías de Campo. Bogotá: Editorial Conservación Internacional Colombia. 478 pp.

Stiles, F. G. 1998. Aves endémicas de Colombia. En Informe nacional sobre el estado de la biodiversidad en Colombia. Tomo 1. Diversidad biológica. Cháves M. E., N. Arango (eds.). Bogotá: Instituto de Investigación de Recursos Biológicos «Alexander von Humboldt», PNUMA, Ministerio del Medio Ambiente. 535 pp.

Verea, C., A. Badillo, A. Solórzano. 2000. Variación en la composición de las comunidades de aves de sotobosque de dos bosques en el norte de Venezuela. Ornitol Neotrop. 11: 65-79. 